\title{
Special Section Guest Editorial: Advances in Gradient Index Optics Technology
}

\author{
Peter L. Marasco, ${ }^{\text {a }}$ Clara Rivero-Baleine, ${ }^{\mathrm{b}}$ Guy Beadie, ${ }^{\mathrm{c}}$ Duncan Moore, ${ }^{\mathrm{d}}$ \\ Kathleen Richardson, ${ }^{\mathrm{e}}$ Michael Ponting, ${ }^{\mathrm{f}}$ and Jasbinder S. Sanghera ${ }^{\mathrm{g}}$ \\ ${ }^{a}$ Air Force Research Laboratory, Wright-Patterson Air Force Base, Ohio, United States \\ ${ }^{b}$ Lockheed Martin Missiles and Fire Control, Grand Prairie, Texas, United States \\ ${ }^{\mathrm{c}}$ Peak Nano, Coppell, Texas, United States \\ ${ }^{\mathrm{d}}$ University of Rochester, Rochester, New York, United States \\ ${ }^{e}$ University of Central Florida, CREOL, Orlando, Florida, United States \\ fPolymerPlus, Valley View, Ohio, United States \\ ${ }^{g}$ U.S. Naval Research Laboratory, Washington, DC, United States
}

Gradient index (GRIN) technology has always held great promise in optical engineering. Refractive lens elements have been based on homogeneous materials for centuries. This has forced much of the physics of light manipulation (optical ray bending) to happen at the surfaces or interfaces between two materials. GRIN materials break this paradigm by enabling the material index of refraction and its related dispersion to change in a controlled, mathematically describable way. This makes the medium more than a way to hold refractive surfaces in space; rather, the medium itself becomes a design degree of freedom for the engineer. If properly used, even an element with two flat surfaces (essentially a plate of glass in the homogenous material context) can add power or aberration corrections to an optical system.

Since their theoretical inception in the 1850s [Maxwell, J.C., Cambridge and Dublin Math. J. 8,188 (1854)], full exploitation of this degree of freedom has remained elusive. For hundreds of years, the goal of most glass manufacturers has been to create homogenous materials for optical systems and instruments. GRIN optics challenges this paradigm by requiring spatially inhomogeneous optical materials. Not only must these optics exhibit spatially varying composition and/or microstructure, but they must do so in specific, mathematically defined ways.

A number of approaches have been devised in the past decades to fabricate useful index gradients-ion exchange and chemical vapor deposition, just to name two. These techniques worked for a number of applications, including endoscopy, the telecommunications industry, and a few others. But all these techniques suffered from some limitation that prevented the extension of GRIN elements to unlimited size (clear aperture), large refractive index gradients (magnitude), and arbitrary (spatial) index profile. To live within these limitations, optical designers and material manufacturers had to engineer around these limitations, often compromising on some other desirable optical function.

Shortly after the turn of the $21^{\text {st }}$ century, things started to change in GRIN technology. Through academic and industrial innovation, fueled initially by government investment, several new material systems, fabrication techniques, and design tools were developed that provided significant advances over traditional approaches and tools. These new techniques offer the potential to overcome a number of limitations, making GRIN useful to a much larger segment of the optics community. These advances include expansion to both bulk and film media, capable of fabricating large-aperture optics, in new broadband materials that extend beyond the visible spectrum of past efforts.

This special section captures the recent advances in these efforts, highlighting cutting edge work occurring in academic and industrial environments. Featured are five papers on various aspects of GRIN technology. We, the contributors and editors of this section, hope you find it useful as you seek to "unleash the power of the GRIN."

(C) 2020 Society of Photo-Optical Instrumentation Engineers (SPIE) 